Hydroécol. Appl. (1993) Tome 5 Vol. 2, pp. 27-42

\title{
Les techniques d'étude de l'ichtyofaune lacustre utilisée en France : bilan et perspectives
}

\section{Technics of study about lakes ichtyofauna in use in France : balance sheet and perspectives}

\section{Degiorgi François*, Guillard Jean ${ }^{\star \star}$, Grandmottet Jean Pierre ${ }^{\star \star \star}$ et Gerdeaux Daniel**}

- Centre d'Etude des Eaux Continentales, Institut des Sciences et Techniques de l'Environnement, Université de Franche Comté, Place Leclerc, 25030 Besancon Cedex

** INRA, Institut de Limnologie, 75, avenue de Corzent, BP 11 F74203 Thonon les Bains

"* Maison Nationale de L'Eau et de la Péche, 25290 Ornans

Résumé. - Cet article présente une revue critique des principales techniques utilisées en France pour échantillonner quantifier ou observer l'ichtyofaune lacustre. Les différents procédés sont classés selon leur mode d'action puis comparés en termes d'avantages et d'inconvénients. Parmi ces dispositifs deux techniques ont bénéficié de progrès récents.

Les filets verticaux à enrouleurs, utilisés suivant le protocole standard adéquat, fournissent, par l'intermédiaire de prises par unité d'effort, des images semi-quantitatives comparable espèce par espèce d'un système à l'autre ou au cours du temps. Cette démarche autorise en outre des analyses habitationnelles assez fines. L'échosondage, qui indique des estimations quantitatives globales tout en permettant de visualiser la répartition spatiale instantanée des poissons en zone centrale, est susceptible d'élargir son champ d'application grâce à des améliorations constantes.

Ces deux procédés montrent une complémentarité intéressante et leur utilisation conjointe offre des perspectives de gain d'informations.

Mots clés. - Ichtyofaune lacustre, échantillonnage, hydroacoustique, filet verticaux, habitat.

Abstract. - This paper consists in a critical review of the main technics of sampling or observation for lakes ichtyofauna in use in France. These procedures are first classified according to their way of working then compared in terms of advantages and disadvantages. Two of these devices have recently progressed.

Vertical gill nets, used according to an adequat standard sampling procedure are able to give catches per unit effort which can be comparable, species by species, to different 
system or during a temporal evolution. Analysis of fish habitat can also be carried out by this approach.

Hydroacoustic, which supplies a quantitative estimation of the biomass and instantaneous visualisations of the distribution of fishes in central zone of lakes, is open to extend its field of applications.

These two procedures show an interesting complementarity and their combined uses will be able to offer good perspectives of gaining informations.

Key words. - Lakes ichtyofauna, sampling method, hydroacoustic, vertical gill nets, fish habitat.

Toute gestion rationnelle des peuplements pisciaires nécessite la connaissance de leur structure et de leur dynamique. D'après Cury (1983), deux démarches peuvent être suivies pour atteindre cet objectif. La première, synthétique, consiste à estimer ou à approcher le stock, par rapport à un référentiel ou dans l'absolu, ou à en déterminer l'évolution au cours du temps. La seconde, analytique, s'attache plutôt à la détermination des différents paramètres de la biologie des populations en présence (croissance, survie, recrutement...) pour déboucher sur le calcul de la production telle que définie par Chapman (1971).

Ces deux approches nécessitent bien évidemment une phase initiale de récolte des données : l'échantillonnage constitue la base de toute méthode de diagnose et apparaît donc particulièrement important. Or dans le cas des plans d'eau, les techniques d'échantillonnage doivent résoudre des problèmes notables dus aux caractéristiques dimensionnelles (étendue et profondeur des systemes) mais aussi structurelles et dynamiques (stratification spécifique à chaque plan d'eau et surtout variable dans le temps) des systèmes considérés. En
France, plusieurs dispositifs d'inventaire et d'observation de l'ichtyofaune lacustre sont actuellement employés en réponse à cette problématique: nous en avons constitué un relevé comparatif en citant les équipes qui les pratiquent le plus couramment.

Parmi ces différents procédés, la technique des filets verticaux et celle de l'échosondage ont bénéficié de progrès récents : elles font donc l'objet d'une présentation plus détaillée. Ces deux dispositifs présentent d'ailleurs une complémentarité intéressante qui sera elle aussi analysée. Leur utilisation conjointe devrait en outre permettre de vérifier ou de recadrer la signification des résultats fournis par chacune d'elles tout en laissant espérer le gain d'un nouveau champ d'information.

\section{SYNOPTIQUIE DES DIFFÉRENTES TECHNIQUES D'APPROCHE DE L'ICHTYOFAUNE LACUSTRE}

\section{I.1. Classification sommaire}

Les différentes techniques utilisées pour appréhender les caractéristiques 
des communautés et/ou des populations pisciaires des systemes d'eau profonde peuvent être classées en fonction du niveau d'information qu'elles fournissent et du mode d'action des dispositifs mis en jeu (cf. figure 1).

Les approches basées sur la capture d'un échantillon ou de l'ensemble du peuplement permettent de réaliser des mesures biométriques (taille, poids, âge...). En revanche, ils induisent pour la plupart, une mortalité importante en ce qui concerne les filets, mineure pour les pièges et les engins actifs en général. Cet aspect est primordial dans le cas de l'utilisation de marquage-recapture : la survie du poisson ne doit pas être hypothéquée lors de la remise à l'eau (Schnabel, 1938; Allen, 1966). Un des principaux biais introduit dans les estimations quantitatives par cette famille de technique basée sur la capture réside dans la sélectivité des engins qui peut concerner la taille, l'espèce ou la localisation des poissons échantilIonnés. La sélectivité de la taille peut être minimisée par l'emploi de batteries d'engins dont les registres de taille se recouvrent (Hamley, 1975; Barbier, 1985; Helser et Condrey, 1991). En revanche la vulnérabilité différentielle des espèces vis à vis de la plupart des engins limite la signification des prises par unité d'effort

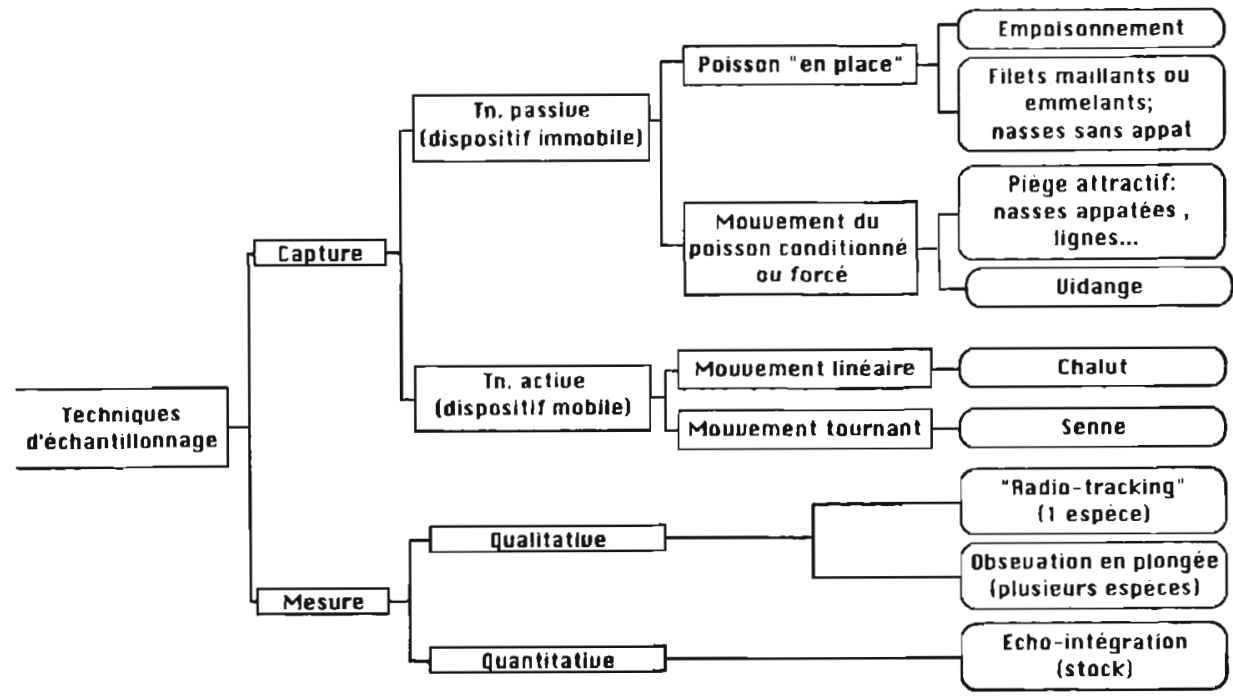

Fig. 1. - Classification schématique des principales techniques utilisées en France pour étudier l'ichtyofaune lacustre.

Fig. 1. - Schematic classification of the main technics used in France for studying ichtyofauna of lakes. 
(PUE) à celle "d'indices d'abondance" qui peuvent être considérés comme proportionnels au stock mais seulement en raisonnant d'un système à l'autre pour comparer les rendements de pêche obtenus pour une espèce donnée (Allen, 1966; Ricker, 1980); certains auteurs utilisent les PUE sous le terme d'abondance "relative" (Legendre et Moreau 1978; Savard et Moreau, 1981...). En outre, il parait important que les moyens d'investigation utilisés puissent l'être dans la totalité des habitats rencontrés afin de satisfaire à la fois à l'homogénéité des engins de capture préconisée par Ricker (1980) et à la prise en compte de la diversité mésologique dans le diagnostic recommandé par Heland (1990).

Contrairement à la plupart de ces dispositifs, l'échosondage ne provoque aucune mortalité. Cependant, lors de l'utilisation de ce procédé, le nombre de mesures biométriques réalisables est limité (cf. §॥-2) tandis que les données obtenues portent sur l'ensemble de la communauté sans différenciation par espèce. Par conséquent, cette approche est le plus souvent couplée à une technique de capture (en général, le chalutage).

Remarquons enfin que l'empoisonnement, le radiotracking ou encore les observations en plongées ne sont, dans le cas des systèmes lacustres, que peu ou pas utilisés en France. La fiabilité des évaluations de stock par vidange n'est pas encore démontrée dans le cas des plans d'eau artificiels de grande taille étant donné les problèmes d'envasement du poisson ain- si que de fuite des juvéniles par les grilles de l'exutoire, et ce dans des proportions difficiles à évaluer. L'utilisation de statistiques de pêche obtenues par enquête est elle aussi encore trés limitée en France (Gerdeaux, 1985).

\section{I.2. Comparaison des différentes techniques}

Les techniques et les modalités d'emploi détaillées des dispositifs les plus utilisés en lac pourront être consultées en lisant:

- Eifac (1975), Ricker (1980) ou Barbier (1985), pour les engins passifs;

- Nelson et Boussu (1974), Léopold et al., (1975) ou Gerdeaux (1985) pour les engins actifs;

- Marchal (1985), Guillard (1991) en ce qui concerne l'échosondage.

La synthèse des indications données par ces auteurs et nos propres observations nous ont permis d'effectuer une comparaison des principales caractéristiques de ces divers procédés, résumées dans le tableau I. Afin de compléter cette information aux gestionnaires sur les techniques d'échantillonnage et d'observations de l'ichtyofaune lacustre, le tableau II dresse la liste non exhaustive des organismes français pratiquant couramment les diverses techniques citées.

Parmi cet ensemble de dispositif, les filets verticaux d'une part et l'échosondage d'autre part ont été sou- 
Tableau I. - Comparaison sommaire des techniques les plus couramment utilisées en France pour l'étude de l'ichtyofaune lacustre.

Table I. - Succinct comparison of the main characteristics of the techniques used in France for the study of the ichtyofauna in lakes.

\begin{tabular}{|c|c|c|c|}
\hline $\begin{array}{l}\text { Caractéristiques } \\
\text { Techniques }\end{array}$ & Avantages & Inconvénients & Remarques \\
\hline Enquête & $\begin{array}{l}\text { "pas de mortalité supplé- } \\
\text { mentaire } \\
\text { *implication directe des } \\
\text { pêcheurs dans la gestion }\end{array}$ & $\begin{array}{l}\text { *données souvent peu } \\
\text { fiables et hétérogènes } \\
\text { *informations limitées }\end{array}$ & $\begin{array}{l}\text { *à utiliser en complé- } \\
\text { ment d'autres } \\
\text { techniques (suivi) }\end{array}$ \\
\hline $\begin{array}{l}\text { Pièges } \\
\text { (nasses) }\end{array}$ & $\begin{array}{l}\text { "pas ou peu de mortalité } \\
\text { "utilisation des mé- } \\
\text { thodes de pêche } \\
\text { "recapture possible pour } \\
\text { estimer les stocks }\end{array}$ & $\begin{array}{l}\text { "peu (ou difficilement) } \\
\text { reproductible } \\
\text { *utilisation } \\
\text { problématique en zone } \\
\text { limnétique (1seule } \\
\text { tranche d'eau prospec- } \\
\text { lée, de hauteur limitée) } \\
\text { "sélectivité importante } \\
\text { (taille et especce) }\end{array}$ & $\begin{array}{l}\text { "standardisation de l'ap- } \\
\text { pât difficile } \\
\text { "possibilité d'utiliser } \\
\text { sans appât pendant la } \\
\text { fraie } \\
\text { "interprétation des résul- } \\
\text { tats difficiles pour les } \\
\text { systemes de grande di- } \\
\text { mension }\end{array}$ \\
\hline Chalut & $\begin{array}{l}\text { "peu de mortalité } \\
\text { "application rapide } \\
\text { "pêche-recapturé } \\
\text { possible }\end{array}$ & $\begin{array}{l}\text { "peu applicable en zone } \\
\text { littorale enherbée } \\
\text { "une seule tranche } \\
\text { d'eau analysée à la fois }\end{array}$ & $\begin{array}{l}\text { "intéressant en complé- } \\
\text { ment de l'échosondage }\end{array}$ \\
\hline Echosondage & $\begin{array}{l}\text { "pas de mortalité induite } \\
\text { "données quantitatives } \\
\text { (biomasse estimée) } \\
\text { "application rapide sur } \\
\text { système de grande } \\
\text { dimension } \\
\text { "observation de toute la } \\
\text { masse d'eau, et de fa- } \\
\text { çon instantanée }\end{array}$ & $\begin{array}{l}\text { "mesures biométriques } \\
\text { encore limitées } \\
\text { "pas d'informations } \\
\text { qualitatives } \\
\text { "observation des es. } \\
\text { pèces benthiques plus } \\
\text { problématiques } \\
\text { "pas d'information sur } \\
\text { les habitats littoraux }\end{array}$ & $\begin{array}{l}\text { "perspectives de progrès } \\
\text { techniques et d'ólargisse- } \\
\text { ment du champ } \\
\text { d'application à court } \\
\text { terme (cf. texte) } \\
\text { "souvent utilisé conjointe- } \\
\text { ment avec une lech- } \\
\text { nique de capture } \\
\text { (chalutage ou filets mail- } \\
\text { lants) }\end{array}$ \\
\hline $\begin{array}{l}\text { Filets } \\
\text { traditionnels }\end{array}$ & $\begin{array}{l}\text { "rapides et fiables pour } \\
\text { sondages qualitatifs } \\
\text { (liste d'espéce) } \\
\text { "indices d'abondance } \\
\text { "nombreuses données } \\
\text { disponibles } \\
\text { "fournit des échantillons } \\
\text { importants pour les } \\
\text { mesures biométriques }\end{array}$ & $\begin{array}{l}\text { "pas de quantitatif absolu } \\
\text { "forte mortalité induite } \\
\text { "approche habitationnelle } \\
\text { difficile } \\
\text { "stratégie d'échantillon- } \\
\text { nage et interprétation } \\
\text { des indices d'abondance } \\
\text { délicate (1 seule } \\
\text { tranche d'eau prospec- } \\
\text { tée à la fois, surface } \\
\text { des nappes différentes } \\
\text { selon la maille) }\end{array}$ & $\begin{array}{l}\text { "nécessité d'utilisation } \\
\text { d'un protocole normalisé } \\
\text { pour pouvoir utiliser les } \\
\text { données }\end{array}$ \\
\hline $\begin{array}{l}\text { Filets } \\
\text { verticaux }\end{array}$ & $\begin{array}{l}\text { "données qualitatives et } \\
\text { indices d'abondance } \\
\text { "faibles mortalités induites } \\
\text { "maniabilité (petites } \\
\text { dimensions) } \\
\text { "échantillonnage simulta- } \\
\text { né de toutes les } \\
\text { tranches d'eau } \\
\text { "approche habitationnelle } \\
\text { "surface des nappes } \\
\text { identique quelque soit } \\
\text { la maille }\end{array}$ & $\begin{array}{l}\text { "pas de quantitatif absolu } \\
\text { "engins complémen- } \\
\text { taires (tramails) } \\
\text { nécessaires pour } \\
\text { certaines espèces } \\
\text { (carpes, anguilles...) } \\
\text { "données comparables } \\
\text { disponibles encore peu } \\
\text { nombreuses }\end{array}$ & $\begin{array}{l}\text { "nécessité d'utilisation } \\
\text { d'un protocole normalisé } \\
\text { pour pouvoir utiliser les } \\
\text { données (proposition } \\
\text { récente d'un tel proto- } \\
\text { cole, cf. texte) } \\
\text { "pourrait être utilisé en } \\
\text { complémentarité avec l'é- } \\
\text { chosondage. } \\
\text { "convient bien à un } \\
\text { suivi régulier }\end{array}$ \\
\hline
\end{tabular}


Tableau I. - Comparaison sommaire des techniques les plus couramment utilisées en France pour l'étude de l'ichtyofaune lacustre.

Table I. - Succinct comparison of the main characteristics of the techniques used in France for the study of the ichtyofauna in lakes.

\section{Caractéristiques Techniques}

Enquête

1

\section{Pièges}

(nasses)

Avantages

\section{Inconvénients}

\section{Remarques}

"pas de mortalité supplé- "données souvent peu mentaire fiables et hétérogènes *implication directe des *informations limitées pêcheurs dans la gestion

"pas ou peu de mortalité "peu (ou difficilement)

"utilisation des mé- reproductible thodes de pêche "utilisation

"recapture possible pour problématique en zone estimer les stocks tranche d'eau prospectée, de hauteur limitée) "sélectivité importante (taille et espèce) limnétique (1seule

*à utiliser en complément d'autres techniques (suivi)

"standardisation de l'appât difficile "possibilité d'utiliser sans appât pendant la fraie *interprétation des résultats difficiles pour les systemes de grande dimension

"peu applicable en zone littorale enherbée "une seule tranche d'eau analysée à la fois

"intéressant en complément de l'échosondage

"applica "pêche-r possible

Echosondage

Filets traditionnels

Filets verticaux "données qualitatives et indices d'abondance "faibles mortalités induites "maniabilité (petites dimensions)

"échantillonnage simultané de toutes les

tranches d'eau

*approche habitationnelle nombreuses

"surface des nappes identique quelque soit la maille 'mesures biométriques encore limitées

"pas d'informations qualitatives

"observation des especes benthiques plus problématiques

"pas d'information sur les habitats littoraux "perspectives de progrès techniques et d'élargissement du champ d'application à court terme (ct. texte)

"souvent utilisé conjointement avec une technique de capture (chalutage ou filets maillants)

-pas de quantitatif absolu "nécessité d'utilisation "forte mortalité induite d'un protocole normalisé "approche habitationnelle pour pouvoir utiliser les difficile donnèes

"stratégie d'échantillonnage et interprétation des indices d'abondance délicate (1 seule tranche d'eau prospectèe à la fois, surface des nappes différentes seion la maille)

"pas de quantitatif absolu "nécessité d'utilisation "engins complémen- d'un protocole normalisé pour pouvoir utiliser les donnèes (proposition récente d'un tel protocole, cf. texte) "pourrait être utilisé en complémentarité avec l'échosondage.

"convient bien à un suivi régulier 
Tableau I. - Comparaison sommaire des techniques les plus couramment utilisées en France pour l'étude de l'ichtyofaune lacustre.

Table I. - Succinct comparison of the main characteristics of the techniques used in France for the study of the ichtyofauna in lakes.

\section{Caractéristiques Techniques}

Enquête

Pièges

(nasses)

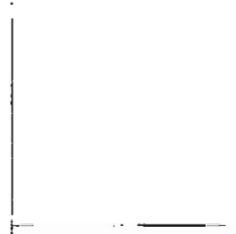

\section{Chalut}

Echosondage

\section{Filets traditionnels}

Filets verticaux

\section{Avantages}

"pas de mortalité supplé- "données souvent peu mentaire -implication directe des *informations limitées pêcheurs dans la gestion

"pas ou peu de mortalité *peu (ou difficilement) "utilisation des mé- reproductible thodes de pêche "utilisation

"recapture possible pour problématique en zone estimer les stocks

trit
tranche d'eau prospectée, de hauteur limitée) "sélectivité importante (taille et espece)

\section{Remarques}

*à utiliser en complément d'autres techniques (suivi)

"standardisation de l'appât difficile

"possibilité d'utiliser sans appât pendant la fraie

*interprétation des résultats difficiles pour les systemes de grande dimension

"peu applicable en zone "intéressant en complélittorale enherbée ment de l'échosondage

"peu de mortalité -application rapide "péche-recapture possible "une seule tranche d'eau analysée à la fois "pas de mortalité induite "mesures biométriques "données quantitatives (biomasse estimée) application rapide sur système de grande dimension

'observation de toute la masse d'eau, et de façon instantanée

\section{encore limitées}

"pas d'informations qualitatives

"observation des espèces benthiques plus problématiques "pas d'information sur les habitats littoraux "perspectives de progrès techniques et d'élargisse-: ment du champ d'application à court terme (cf. texte)

"souvent utilisé conjointe-1 ment avec une tech-

nique de capture (chalutage ou filets maillants)

"rapides et fiables pour sondages qualitatifs (liste d'espèce) "indices d'abondance *nombreuses données disponibles

fournit des échantillons importants pour les mesures biométriques

"pas de quantitatif absolu "forte mortalité induite "approche habitationnelle difficile

"stratégie d'échantillonnage et interprétation des indices d'abondance délicate ( 1 seule tranche d'eau prospectée à la fois, surface des nappes différentes selon la maille)

"données qualitatives et indices d'abondance *aables mortalites induites "maniabilité (petites dimensions) "échantillonnage simultané de toutes les tranches d'eau approche habitationnelle nombreuses

"pas de quantitatif absolu "nécessité d'utilisation "engins complémen. taires (tramails) nécessaires pour certaines espèces (carpes, anguilles...) "données comparables disponibles encore peu "surface des nappes identique quelque soit la maille d'un protocole normalisé pour pouvoir utiliser les données (proposition récente d'un tel protocole, cf. texte) "pourrait être utilisé en complémentarité avec l'échosondage.

"Convient bien à un suivi régulier 
Tableau II. - Liste non exhaustive d'organismes français pratiquant couramment une ou plusieurs techniques d'étude de l'ichtyofaune lacustre.

Table II. - Non exhaustive list of french organizations which usually practise one or several methods for the study of lake ichtyofauna.

\begin{tabular}{|c|c|c|c|c|c|c|c|}
\hline $\begin{array}{l}\text { Techniques } \\
\text { Organismes }\end{array}$ & $\begin{array}{c}\text { Enquête } \\
\text { pêche à la ligne } \\
\text { ou professionnelle }\end{array}$ & Piège & Seine & Chalut & $\begin{array}{l}\text { Echo- } \\
\text { son- } \\
\text { dage }\end{array}$ & $\begin{array}{l}\text { Filets } \\
\text { traditionnels }\end{array}$ & $\begin{array}{c}\text { Filets } \\
\text { verticaux }\end{array}$ \\
\hline CSP & $x x$ & $X X X$ & $x$ & $x$ & & $x x$ & $x$ \\
\hline $\begin{array}{c}\text { INRA } \\
\text { Thonon les Bains }\end{array}$ & $x x$ & $x$ & & $x$ & $x \times x$ & $x$ & \\
\hline $\begin{array}{l}\text { ISTEE } \\
\text { Besançon }\end{array}$ & $x$ & $x$ & & & & $x x$ & $x x x$ \\
\hline $\begin{array}{l}\text { MNEP } \\
\text { Ornans }\end{array}$ & $x$ & $x$ & & & & $x$ & $x x x$ \\
\hline $\begin{array}{c}\text { SRAE } \\
\text { Rhône-Alpes }\end{array}$ & $x$ & & & & & $x x$ & $x$ \\
\hline $\begin{array}{l}\text { Université } \\
\text { Toulouse }\end{array}$ & & & & & $x \times x$ & $x X$ & \\
\hline $\begin{array}{l}\text { ENSAT } \\
\text { Toulouse }\end{array}$ & & $x x x$ & & & & $x x x$ & \\
\hline $\begin{array}{l}\text { Université } \\
\text { Limoges }\end{array}$ & & $x$ & $x x$ & & & $x x$ & \\
\hline $\begin{array}{l}\text { Universite } \\
\text { Metz }\end{array}$ & $x$ & & & & & $x$ & $x$ \\
\hline $\begin{array}{c}\text { CEMAGREF } \\
\text { (Paris, Montpellier, } \\
\text { Aix en provence, } \\
\text { Bordeaux...) }\end{array}$ & $x$ & $x$ & & & & $x x$ & \\
\hline
\end{tabular}

mis récemment à de nouvelles expérimentations qui ont permis de proposer de meilleures modalités et conditions d'application afin d'optimiser la précision des résultats obtenus et la fiabilité des inter- prétations qui en découlent (CEEC, 1988; Iste, 1992; Gerdeaux et Guillard, 1992: Degiorgi et Grandmottet, 1993). Nous avons donc choisi de développer la présentation de ces deux approches. 
Tableau II. - Liste non exhaustive d'organismes français pratiquant couramment une ou plusieurs techniques d'étude de l'ichtyofaune lacustre.

Table II. - Non exhaustive list of french organizations which usually practise one or several methods for the study of lake ichtyofauna.

\begin{tabular}{|c|c|c|c|c|c|c|c|}
\hline $\begin{array}{l}\text { Techniques } \\
\text { Organismes }\end{array}$ & $\begin{array}{c}\text { Enquête } \\
\text { péche a la ligne } \\
\text { ou professionnelle }\end{array}$ & Piège & Seine & Chalut & $\begin{array}{l}\text { Echo- } \\
\text { son- } \\
\text { dage }\end{array}$ & $\begin{array}{c}\text { Filets } \\
\text { traditionnels }\end{array}$ & $\begin{array}{c}\text { Filets } \\
\text { verticaux }\end{array}$ \\
\hline CSP & $x x$ & $x x x$ & $x$ & $x$ & & $x x$ & $x$ \\
\hline $\begin{array}{c}\text { INRA } \\
\text { Thonon les Bains }\end{array}$ & $x x$ & $x$ & & $x$ & $x x x$ & $x$ & \\
\hline $\begin{array}{c}\text { ISTEE } \\
\text { Besançon }\end{array}$ & $x$ & $x$ & & & & $x x$ & $x X X$ \\
\hline $\begin{array}{l}\text { MNEP } \\
\text { Ornans }\end{array}$ & $x$ & $x$ & & & & $x$ & $x x x$ \\
\hline $\begin{array}{c}\text { SRAE } \\
\text { Rhóne-Alpes }\end{array}$ & $x$ & & & & & $x x$ & $x$ \\
\hline $\begin{array}{l}\text { Universite } \\
\text { Toulouse }\end{array}$ & & & & & $x X x$ & $x x$ & \\
\hline $\begin{array}{l}\text { ENSAT } \\
\text { Toulouse }\end{array}$ & & $x x x$ & & & & $x x x$ & \\
\hline $\begin{array}{l}\text { Universite } \\
\text { Limoges }\end{array}$ & & $x$ & $x x$ & & & $x x$ & \\
\hline $\begin{array}{l}\text { Université } \\
\text { Metz }\end{array}$ & $x$ & & & & & $x$ & $x$ \\
\hline $\begin{array}{c}\text { CEMAGREF } \\
\text { (Paris, Montpellier, } \\
\text { Aix en provence. } \\
\text { Bordeaux...) }\end{array}$ & $x$ & $x$ & & & & $x x$ & \\
\hline
\end{tabular}

mis récemment à de nouvelles expérimentations qui ont permis de proposer de meilleures modalités et conditions d'application afin d'optimiser la précision des résultats obtenus et la fiabilité des inter- prétations qui en découlent (CEEC, 1988 ; Iste, 1992 ; Gerdeaux et Guillard, 1992: Degiorgi et Grandmottet, 1993). Nous avons donc choisi de développer la présentation de ces deux approches. 
Tableau II. - Liste non exhaustive d'organismes français pratiquant couramment une ou plusieurs techniques d'étude de l'ichtyofaune lacustre.

Table II. - Non exhaustive list of french organizations which usually practise one or several methods for the study of lake ichtyofauna.

\begin{tabular}{|c|c|c|c|c|c|c|c|}
\hline $\begin{array}{l}\text { Techniques } \\
\text { Organismes }\end{array}$ & $\begin{array}{c}\text { Enquête } \\
\text { pêche a la ligne } \\
\text { ou professionnelle }\end{array}$ & Piège & Seine & Chalut & $\begin{array}{l}\text { Echo- } \\
\text { son- } \\
\text { dage }\end{array}$ & $\begin{array}{c}\text { Filets } \\
\text { traditionnels }\end{array}$ & $\begin{array}{c}\text { Filets } \\
\text { i verticaux }\end{array}$ \\
\hline - & 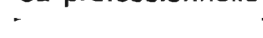 & & & $-\ldots$ & & $\ldots$ & .. \\
\hline CSP & $x x$ & $x x x$ & $x$ & $x$ & & $x x$ & $x$ \\
\hline $\begin{array}{c}\text { INRA } \\
\text { Thonon les Bains }\end{array}$ & $x x$ & $x$ & & $x$ & $x x x$ & $x$ & \\
\hline $\begin{array}{c}\text { ISTEE } \\
\text { Besançon }\end{array}$ & $x$ & $x$ & & & & $x x$ & $x x x$ \\
\hline $\begin{array}{l}\text { MNEP } \\
\text { Ornans }\end{array}$ & $\mathbf{x}$ & $x$ & & & & $x$ & $x X X$ \\
\hline $\begin{array}{l}\text { SRAE } \\
\text { Rhône-Alpes }\end{array}$ & $x$ & & & & & $x x$ & $x$ \\
\hline $\begin{array}{l}\text { Université } \\
\text { Toulouse }\end{array}$ & & & & & $x x x$ & $x x$ & \\
\hline $\begin{array}{l}\text { ENSAT } \\
\text { Toulouse }\end{array}$ & & $x x x$ & & & & $x x x$ & \\
\hline $\begin{array}{l}\text { Université } \\
\text { Limoges }\end{array}$ & & $x$ & $x x$ & & & $x x$ & \\
\hline $\begin{array}{l}\text { Université } \\
\text { Metz }\end{array}$ & $x$ & & & & & $x$ & $x$ \\
\hline $\begin{array}{c}\text { CEMAGREF } \\
\text { (Paris, Montpellier, } \\
\text { Aix en provence, } \\
\text { Bordeaux...) }\end{array}$ & ! & $x$ & & & & $x x$ & \\
\hline . & . & & & $\cdot$ & & & $\cdot$ \\
\hline $\begin{array}{l}X: \text { simple possibilite } \\
X X: \text { utilisation cour } \\
X X X\end{array}$ & $\begin{array}{l}\text { té d'application ou ut } \\
\text { rante }\end{array}$ & tilisation & récen & & & & \\
\hline
\end{tabular}

mis récemment à de nouvelles expérimentations qui ont permis de proposer de meilleures modalités et conditions d'application afin d'optimiser la précision des résultats obtenus et la fiabilité des inter- prétations qui en découlent (CEEC, 1988 ; Iste, 1992 ; Gerdeaux et Guillard, 1992; Degiorgi et Grandmottet, 1993). Nous avons donc choisi de développer la présentation de ces deux approches. 


\section{MODALITÉ ET CONDITIONS D'UTILISATION DE DEUX TECHNIQUES D'OBSERVATION ET D'ÉCHANTILLONNAGE DE L'ICHTYOFAUNE LACUSTRE}

\section{II.1. Les filets verticaux}

Les avantages pratiques et la spécificité de ce dispositif d'inspiration américaine (Horak et Tanner, 1964), sont présentés et développés par Grandmottet et Vaudaux (1989); Guyard et al. (1989); Iste (1992). L'utilisation des filets verticaux à enrouleurs depuis 1986 sur une dizaine de lacs, retenues artificielles et systèmes potamiques a permis l'élaboration d'un protocole d'échantillonnage standard fournissant une image stable et comparable de la communauté étudiée ${ }^{(1)}$. Ces filets, de faible largeur $(2 \mathrm{~m})$ et de hauteur modulable (de 0,50 à $100 \mathrm{~m}$ ), présentent de nombreux avantages par rapport aux filets traditionnels: en particulier, ils sont d'une grande maniabilité, échantillonnent toute la tranche d'eau quel que soit le site où ils sont disposés (pour des lacs de profondeur maximum inférieure à $100 \mathrm{~m}$ ) et occasionnent des mortalités pisciaires réduites; aussi se prêtent-ils particulièrement bien à la prospection systématique du volume lacustre et autorisent-ils la réalisation de réplicats.

L'effort de pêche unitaire est constitué d'une batterie de 6 filets verticaux

(1) Programme financé par le SRETIE el le Conseil Supérieur de la Pêche. de taille de maille variant de 10 à $60 \mathrm{~mm}$ par pas de $10 \mathrm{~mm}$, disposée sur un poste déterminé pendant 24 heures. Auparavant, l'espace lacustre doit avoir été divisé en compartiments d'attraction différentielle en suivant un schéma descriptif directif basé sur la topographie aquatique (morphologie de la cuvette, substrat et support, hauteur d'eau...) et dont la validité a été démontrée a posteriori (Iste, 1992; Degiorgi et Grandmottet, 1993): les différentes espèces réalisent en effet des arrangements spatiaux dynamiques mais conditionnés par les pôles d'attraction pré-définis. Une cartographie de ces pôles d'attraction de la cuvette lacustre ayant été dressée, tous les compartiments sont alors prospectés simultanément au cours d'un effort " global » appelé aussi séquence qui est répété plusieurs fois pour chaque campagne saisonnière (Iste, op. cité).

L'analyse des séries de prise par effort global montre que la stabilité numérique espèce par espèce et même pondérale toutes espèces confondues est atteinte avec une précision de moins de $20 \%$ au bout de trois séquences successives, et ce pour la plupart des espèces rencontrées au cours des expérimentations menées jusqu'ici (Iste, op. cité; Degiorgi et Grandmottet, Bull. Fr. de Pisc, soumis); cette stabilisation des résultats est illustrée par 3 exemples présentés sur la figure 2. La reproductibilité de cette approche permet donc de comparer des systèmes entre eux ou de suivre dans le temps l'évolution d'un peuplement donné. 


\section{MODALITÉ ET CONDITIONS D'UTILISATION DE DEUX TECHNIQUES D'OBSERVATION ET D'ÉCHANTILLONNAGE DE L'ICHTYOFAUNE LACUSTRE}

\section{II.1. Les filets verticaux}

Les avantages pratiques et la spécificité de ce dispositif d'inspiration américaine (Horak et Tanner, 1964), sont présentés et développés par Grandmottet et Vaudaux (1989); Guyard et al. (1989); Iste (1992). L'utilisation des filets verticaux à enrouleurs depuis 1986 sur une dizaine de lacs, retenues artificielles et systèmes potamiques a permis l'élaboration d'un protocole d'échantillonnage standard fournissant une image stable et comparable de la communauté étudiée ${ }^{(1)}$. Ces filets, de faible largeur $(2 \mathrm{~m}$ ) et de hauteur modulable (de 0,50 à $100 \mathrm{~m}$ ), présentent de nombreux avantages par rapport aux filets traditionnels: en particulier, ils sont d'une grande maniabilité, échantillonnent toute la tranche d'eau quel que soit le site où ils sont disposés (pour des lacs de profondeur maximum inférieure à $100 \mathrm{~m}$ ) et occasionnent des mortalités pisciaires réduites; aussi se prêtent-ils particulièrement bien à la prospection systématique du volume lacustre et autorisent-ils la réalisation de réplicats.

L'effort de pêche unitaire est constitué d'une batterie de 6 filets verticaux

(1) Programme financé par le SRETIE et le Conseil Supérieur de la Pêche. de taille de maille variant de 10 à $60 \mathrm{~mm}$ par pas de $10 \mathrm{~mm}$, disposée sur un poste déterminé pendant 24 heures. Auparavant, l'espace lacustre doit avoir été divisé en compartiments d'attraction différentielle en suivant un schéma descriptif directif basé sur la topographie aquatique (morphologie de la cuvette, substrat et support, hauteur d'eau...) et dont la validité a été démontrée a posteriori (Iste, 1992; Degiorgi et Grandmottet, 1993) : les différentes espèces réalisent en effet des arrangements spatiaux dynamiques mais conditionnés par les pôles d'attraction pré-définis. Une cartographie de ces pôles d'attraction de la cuvette lacustre ayant été dressée, tous les compartiments sont alors prospectés simultanément au cours d'un effort "global" appelé aussi séquence qui est répété plusieurs fois pour chaque campagne saisonnière (Iste, op. cité).

L'analyse des séries de prise par effort global montre que la stabilité numérique espèce par espèce et même pondérale toutes espèces contondues est atteinte avec une précision de moins de $20 \%$ au bout de trois séquences successives, et ce pour la plupart des espèces rencontrées au cours des expérimentations menées jusqu'ici (Iste, op. cité; Degiorgi et Grandmottet, Bull. Fr. de Pisc, soumis); cette stabilisation des résultats est illustrée par 3 exemples présentés sur la figure 2 . La reproductibilité de cette approche permet donc de comparer des systèmes entre eux ou de suivre dans le temps l'évolution d'un peuplement donné. 


\section{MODALITÉ ET CONDITIONS D'UTILISATION DE DEUX TECHNIQUES D'OBSERVATION ET D'ÉCHANTILLONNAGE DE L'ICHTYOFAUNE LACUSTRE}

\section{II.1. Les filets verticaux}

Les avantages pratiques et la spécificité de ce dispositif d'inspiration américaine (Horak et Tanner, 1964), sont présentés et développés par Grandmottet et Vaudaux (1989); Guyard et al. (1989); Iste (1992). L'utilisation des filets verticaux à enrouleurs depuis 1986 sur une dizaine de lacs, retenues artificielles et systèmes potamiques a permis l'élaboration d'un protocole d'échantillonnage standard fournissant une image stable et comparable de la communauté étudiée ${ }^{(1)}$. Ces filets, de faible largeur $(2 \mathrm{~m})$ et de hauteur modulable (de 0,50 à $100 \mathrm{~m}$ ), présentent de nombreux avantages par rapport aux filets traditionnels: en particulier, ils sont d'une grande maniabilité, échantillonnent toute la tranche d'eau quel que soit le site où ils sont disposés (pour des lacs de profondeur maximum inférieure à $100 \mathrm{~m}$ ) et occasionnent des mortalités pisciaires réduites; aussi se prêtent-ils particulièrement bien à la prospection systématique du volume lacustre et autorisent-ils la réalisation de réplicats.

L'effort de pêche unitaire est constitué d'une batterie de 6 filets verticaux

(1) Programme financé par le SRETIE et le Conseil Supérieur de la Pêche. de taille de maille variant de 10 à $60 \mathrm{~mm}$ par pas de $10 \mathrm{~mm}$, disposée sur un poste déterminé pendant 24 heures. Auparavant, l'espace lacustre doit avoir été divisé en compartiments d'attraction différentielle en suivant un schéma descriptif directif basé sur la topographie aquatique (morphologie de la cuvette, substrat et support, hauteur d'eau...) et dont la validité a été démontrée a posteriori (Iste, 1992; Degiorgi et Grandmottet, 1993): les différentes espèces réalisent en effet des arrangements spatiaux dynamiques mais conditionnés par les pôles d'attraction pré-définis. Une cartographie de ces pôles d'attraction de la cuvette lacustre ayant été dressée, tous les compartiments sont alors prospectés simultanément au cours d'un effort "global» appelé aussi séquence qui est répété plusieurs fois pour chaque campagne saisonnière (Iste, op. cité).

L'analyse des séries de prise par effort global montre que la stabilité numérique espèce par espèce et même pondérale toutes espèces confondues est atteinte avec une précision de moins de $20 \%$ au bout de trois séquences successives, et ce pour la plupart des espèces rencontrées au cours des expérimentations menées jusqu'ici (Iste, op. cité; Degiorgi et Grandmottet, Bull. Fr. de Pisc, soumis); cette stabilisation des résultats est illustrée par 3 exemples présentés sur la figure 2. La reproductibilité de cette approche permet donc de comparer des systèmes entre eux ou de suivre dans le temps l'évolution d'un peuplement donné. 

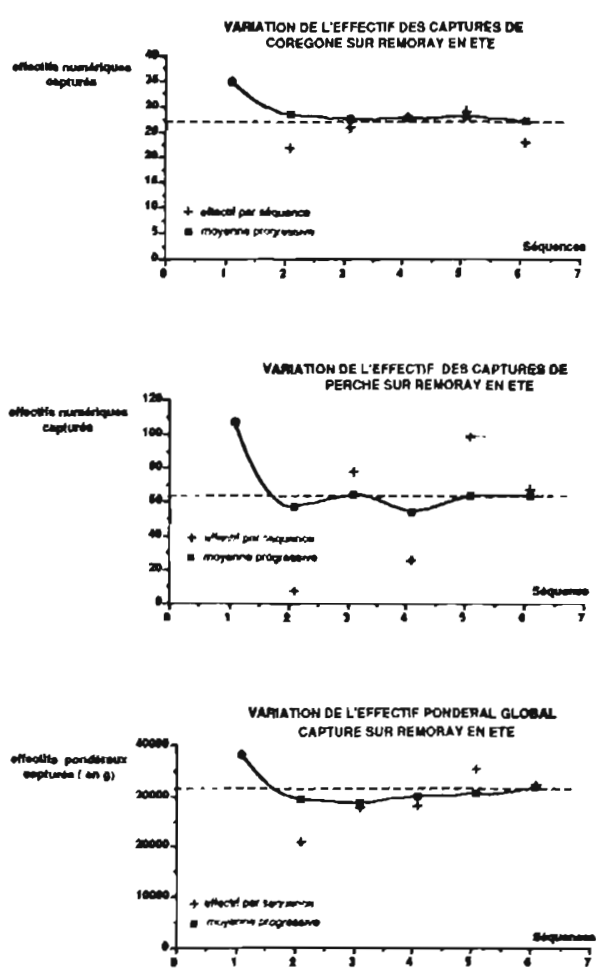

Fig. 2. - Stabilisation des résultats obtenus par répétition de l'effort de pêche global standard (Iste, 1992).

Fig. 2. - Statistical stabilisations of the results obtained by repetition of standard fishing effort (Iste, 1992).

Par ailleurs la différence d'activité du poisson et la dynamique des populations (en particulier en ce qui concerne le recrutement des premières classes maillables) justifient l'intérêt d'appliquer ce protocole de façon tri-saisonnière. La comparaison, sur la figure 3, des structures des échantillons de perches (Perca fluviatilis) capturées dans la Retenue du

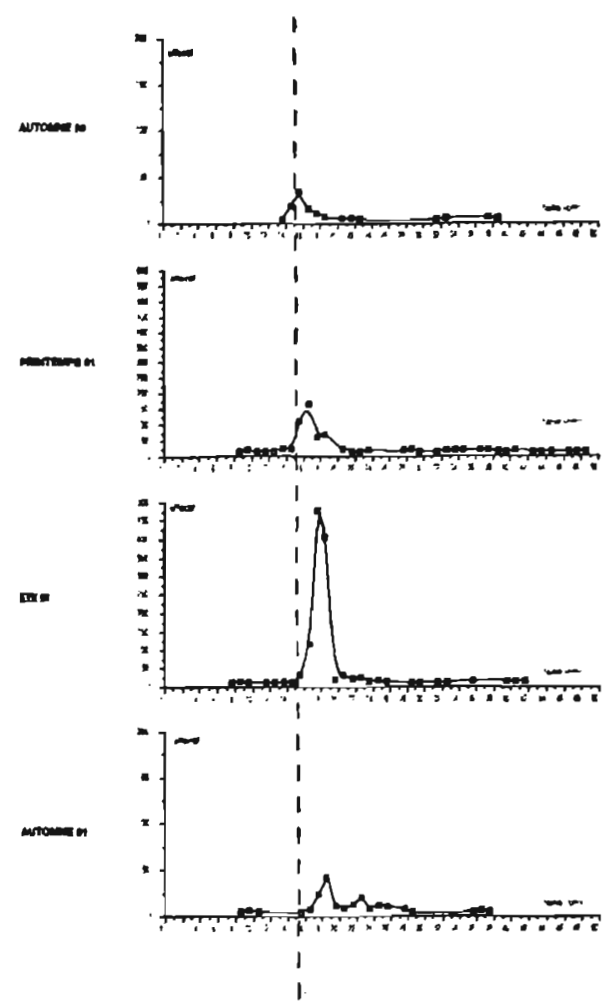

Fig. 3. - Structure taille-fréquence des échantillons saisonniers de perches (Perca fluviatilis) capturés sur le Vieux pré au moyen d'un protocole standard d'échantiitonnage par filets verticaux (MNEP, 1992).

Fig. 3. - Size-frequence structure of the samples of Perch (Perca fluviatilis) caught in the artificial pond of "Vieux Pré" (1990-1991) with vertical gill-nets used according to a standard sampling procedure (MNEP, 1992).

Vieux Pré (Meurthe et Moselle, 450 ha pour 65 mètres de profondeur maximale) au cours de 4 campagnes saisonnières successives illustre ce propos et met en évidence la vitesse de croissance des premières classes d'âge et les problèmes périodiques de 
recrutement induits par les marnages pour cette espèce (MNEP, 1992) (2).

\section{Le protocole proposé présente} également l'avantage de fournir des observations fiables et précises sur les variations spatio-temporelles de la fréquentation des compartiments qui réalisent une partition de l'espace lacustre. A titre d'exemple, la figure 4 montre la différence d'attractivité des compartiments littoraux de la Retenue du Vieux Pré observés durant les 4 campagnes réalisées. D'après cette approche, les fonds nus s'avèrent nettement moins hospitaliers que les compartiments présentant un support végétal quel qu'il soit, en particulier les arbres immergés dans la retenue pour chacune des saisons considérées et les hélophytes ou les prairies immergées au printemps. On constate également la différence d'attractivité des afférences entre l'automne 90, où le niveau du plan d'eau était très bas et où les sources périphériques constituaient un vecteur hydrologique sensible dans la retenue, et l'automne 91 durant lequel ces afférences étaient submergées ou à fleur d'eau et par conséquent moins attractives (MNEP, op. cit.). De la même façon, les images successives obtenues sur la répartition des espèces pélagiques permettent de relier le fonctionnement du système à son potentiel de production piscicole. Sur la figure 5, on peut ainsi observer la limitation de l'espace utilisable pour les Corégones du lac de Remoray (Jura) par suite

(2) L'étude du peuplement pisciaire de la retenue du Vieux Pré a été financée par EDF.

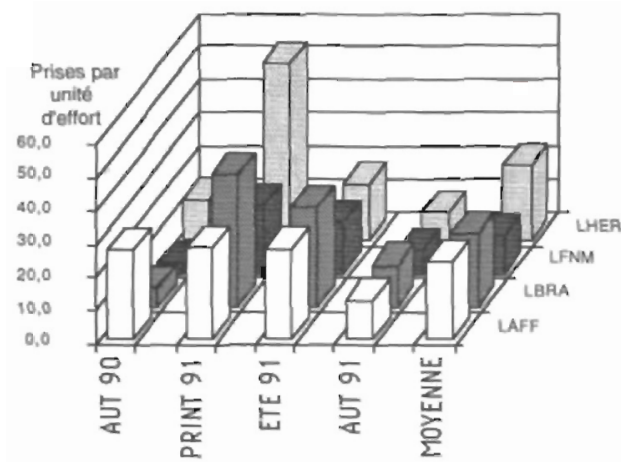

LEGENDE

LAFF : aftérences
LBRA : branches ou souches immergees
LFNM : fonds nus ou decapes
LHER : hélophytes ou prairies immergées.

Fig. 4. - Comparaison des prises par unité d'effort (toutes espèces confondues) réalisées sur les différents compartiments littoraux de la Retenue du Vieux Pré

Fig. 4. - Comparison of catches by unit effort (all species together) in the different littoral habitats of "Vieux Prè " pond.

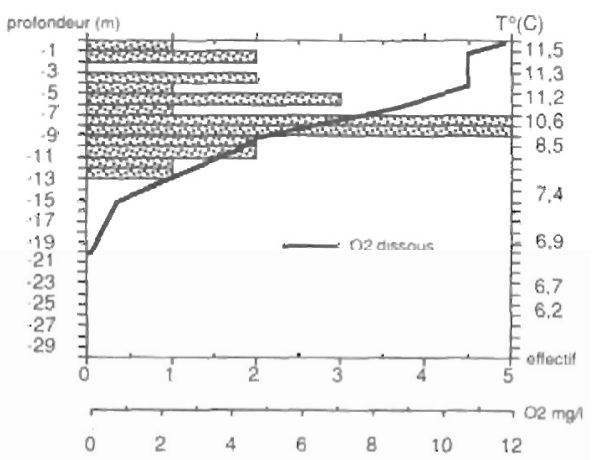

Fig. 5. - Limitation de l'espace disponible pour les corégones (Corégonus sp. la Palée) par la désoxygénation de l'eau de fond (Remoray, Octobre 1990); la tempéraiure des différentes strates de profondeur est directement indiquée par l'échelle verticale de droite.

Fig. 5. - Limitation of space available for white fish (Coregonus sp. la Palée) due to desoxygenation of bottom water (lake of Remoray; October 1990); the temperature of the different strata of depth is directly precised on the scale of the right side. 
de la désoxygénation estivale des fonds (voir aussi Degiorgi et Grandmottet 1992).

Parallèlement aux avantages notables du protocole proposé, il convient d'observer qu'il ne fournit que des indices d'abondance sous forme de PUE et qu'il ne permet pas d'observer les différences de localisations des espèces entre le jour et la nuit, puisque les filets ne sont relevés qu'une fois par intervalle de 24 heures.

\section{II.2. L'hydro-acoustique}

Le principe de l'hydro-acoustique est basé sur la diffusion des ondes sonores dans l'eau, et par leurs réflexions sur les obstacles rencontrés, en particulier les poissons. On démontre de façon générale que l'intensité de l'écho d'un poisson dépend de sa biomasse (Marchal, 1985). Le bateau parcourant un trajet sur une étendue d'eau, le signal acoustique est émis à intervalles réguliers; on obtient ainsi pour toute la strate d'eau des densités de poissons qu'il est possible par la suite de cartographier et d'estimer (figure 6). Seule la frange vraiment littorale reste inaccessible, ainsi que le $1^{\text {er }}$ mètre en dessous de la surface, mais il est possible d'échantillonner dans des zones où le fond est inférieur à 4 mètres (Gerdeaux, 1986; Gerdeaux et Guillard, 1992).

Le simple traitement graphique des données permet de mettre en évidence les variations de la structure spatiale du peuplement pisciaire dans un lac, tant dans la dimension hori- zontale que verticale (figure 7). Les campagnes doivent être effectuées pendant un laps de temps suffisamment court pour ne pas recouper des périodes de natures différentes, et permettent ainsi d'avoir une vision instantanée de la répartition du peuplement pisciaire d'un lac. En particulier entre les périodes diurnes et nocturnes les poissons effectuent des migrations importantes. De jour ils sont souvent posés sur le fond et en banc, et au crépuscule se dispersent et migrent vers des strates supérieures.

A partir de ces estimations et grâce à l'utilisation de techniques statistiques qui prennent en compte les caractéristiques des données (échantillonnage en continu et variables dépendantes spatialement) on peut cartographier une étendue d'eau et obtenir un estimateur de la biomasse totale de poisson. La couverture nocturne d'un lac de moins de 5000 ha peut être réalisée en moins de 6 heures avec une densité d'échantillonnage suffisante.

Avec les précautions d'emploi nécessaires on possède un outil fiable, rapide, de description et d'évaluation des peuplements pisciaires pélagiques lacustres. L'échosondage permet un échantillonnage identique des différentes espèces et des différentes cohortes, à partir du seuil de détection qui est de l'ordre de $2-3 \mathrm{~cm}$ avec les sondeurs traditionnels utilisés en eau douce (fréquence $70 \mathrm{kHz}$ ). En répétant l'opération à des périodes différentes on peut mesurer l'évolution des stocks, et de leurs répartitions spatiales tant saisonnières (Guillard et al., 


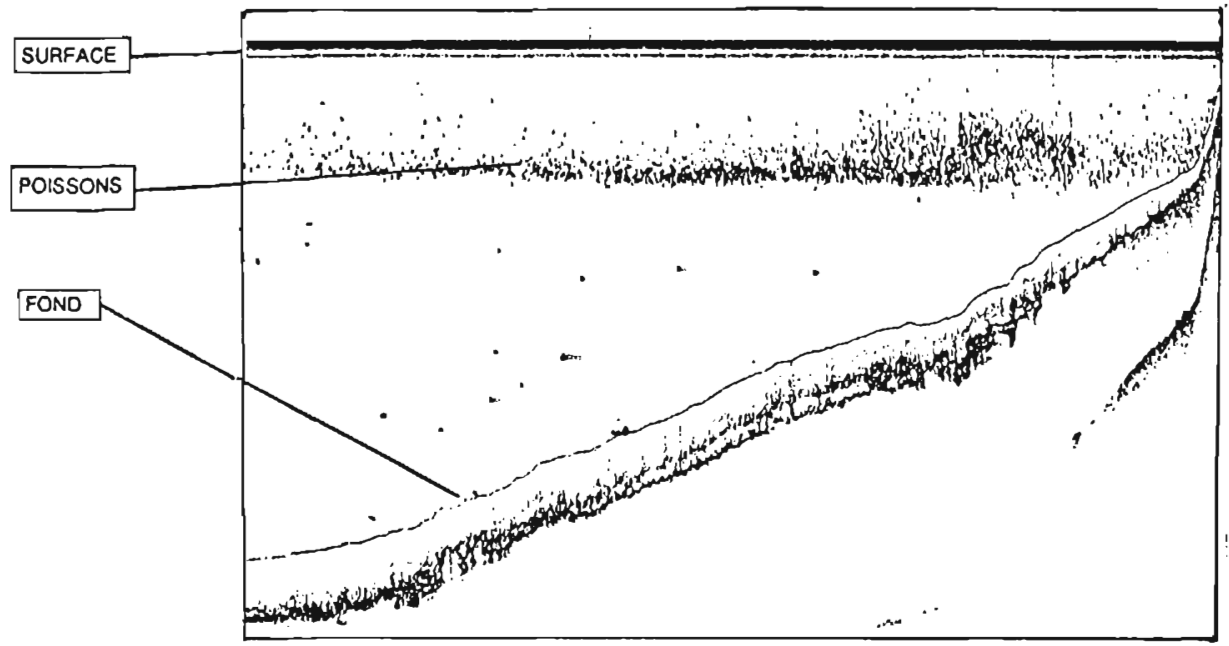

Fig. 6. - Echogramme indiquant une répartition des poissons serrée au niveau de la thermocline et une concentration littorale importante (Annecy, Octobre 1988).

Fig. 6. - Echogram showing the location of fish near the level of thermoclin and an important littoral concentration (lake of Annecy, October 1988).

1992) qu'annuelles (Guillard, 1991). Les évolutions technologiques permettent d'obtenir du matériel de plus en plus performant et de plus en pratique à utiliser (miniaturisation). De plus si l'on peut déjà obtenir dans certaines conditions à partir de sondeur traditionnel une estimation en classe de taille des poissons (Lindem, 1983; Guillard et Gerdeaux, soumis), les nouveaux sondeurs dits à "faisceau éclaté " permettent en recevant en plusieurs points proches les échos réfléchis de repositionner le poisson dans l'espace et de déterminer ainsi sa taille réelle par une analyse complexe des décalages de phase. Ces informations complémentaires permettent d'affiner les résultats obtenus sur le niveau des stocks, sur leurs répartitions dans l'espace, et leurs évolutions.

\section{COMPLÉMENTARITÉ DES DEUX TECHNIQUES}

L'utilisation conjointe de l'échosondage et de filets maillants traditionnels a déjà été pratiquée par plusieurs auteurs (Dembinski, 1971; Sandlund et al., 1985) qui ont employé des engins de capture traditionnels. L'un des intérêts majeurs de telles opérations consiste à obtenir à la fois une image qualitative et semiquantitative grâce aux filets maillants et une évaluation quantitative globale fournie par l'hydroacoustique; il reste alors à établir des liens rationnels en- 


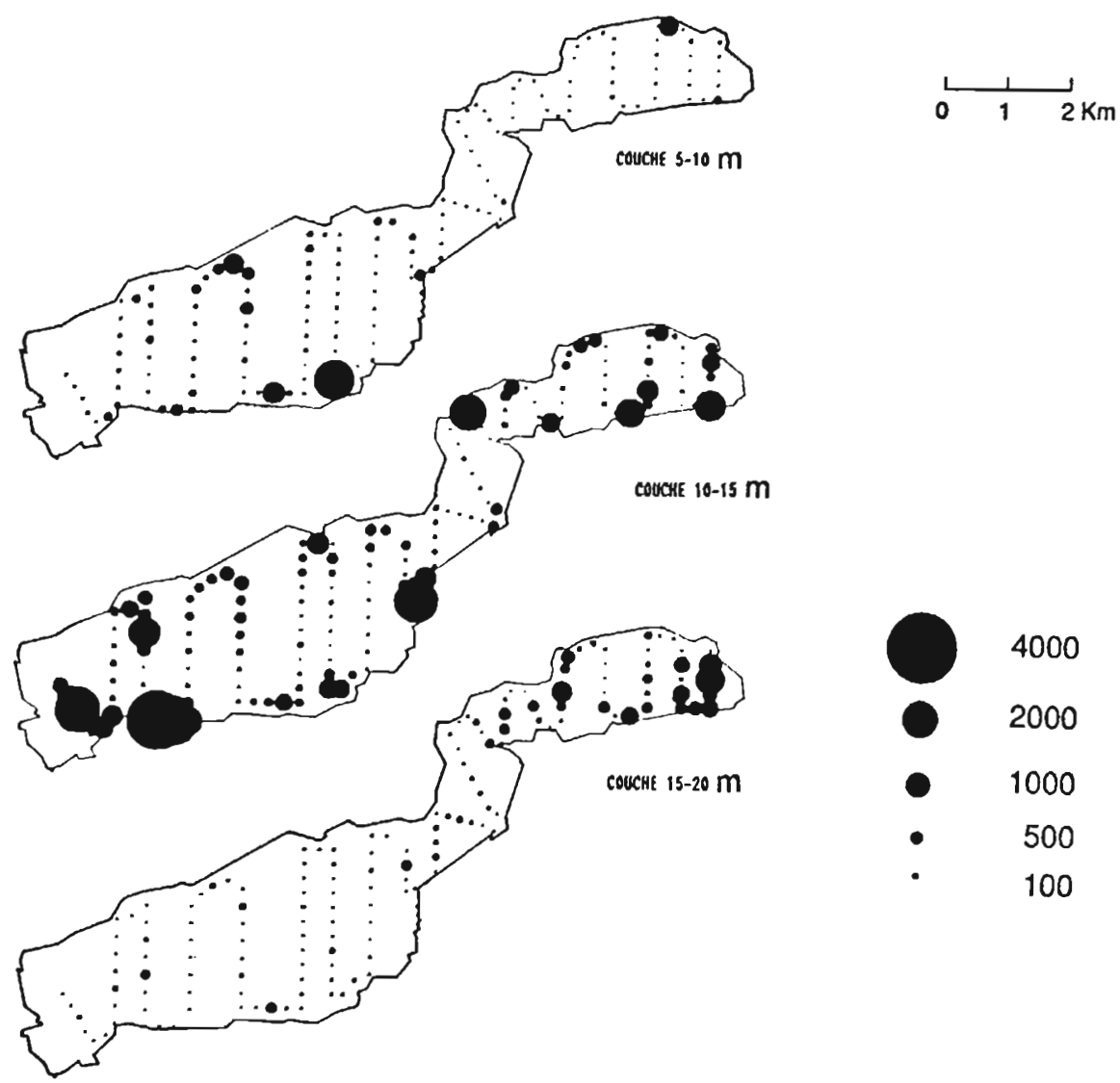

NORD

Fig. 7. - Cartographie d'une campagne d'échointégration réalisée sur le lac d'Annecy; les résultat des séquences d'échointégration de $2 \mathrm{mn}$ sont représentés par des cercles de rayon proportionnel au nombre d'unités d'échointégration enregistrées, et ce pour 3 couches d'eau différentes.

Fig. 7. - Mapping (for three differents layers) of an echointegration survey made on lake of Annecy. The results of echointegration sequences of two minutes are represented by circles the radius of which are proportional to the number of integrated echos recorded.

tre les deux niveaux d'information. Pour que cette démarche soit justifiée, il convient de vérifier d'abord que les PUE utilisées pour ce raisonnement sont effectivement comparables entre elles c'est à dire obtenues au moyen d'un protocole standard et reproductible. Par ailleurs, les filets classiques présentent l'inconvénient de n'échantillonner qu'une tranche d'eau limitée (Barbier 1985, Guyard et al., 1989). La confrontation de la localisation des 
captures au filet, identifiées à l'espèce, et de la position des cibles déterminées par l'hydroacoustique risque donc de se heurter à des lacunes d'information qui rendent les interprétations difficiles voire hasardeuses. En revanche les filets verticaux échantillonnent simultanément toute la tranche d'eau et peuvent être disposés sur un ensemble de compartiments qui réalisent une partition de l'espace lacustre; la confrontation de l'image de la répartition des espèces avec les échogrammes réalisés pendant la même période est alors plus facile et plus fiable (cf. fig. 8, à comparer avec la figure 6).

II convient toutefois de ne pas oublier que l'échosondage indique une localisation à un moment donné, en général nocturne, tandis que les filets échantillonnent sur un nycthémère. Les similarités ou les écarts éventuels entre les deux points de vue constitueraient d'ailleurs des observations supplémentaires intéressantes quant à l'organisation spatio-temporelle de l'ichtyofaune lacustre. Quoiqu'il en soit, la confrontation des résultats obtenus par les deux techniques devrait permettre de préciser ou de recadrer les interprétations qui en découlent. Ainsi, si on admet que les indices d'abondance constitués par les PUE espèce par espèce sont au moment de l'échantillonnage, proportionnels au stock de chacune des populations concernées (Ricker, 1980), il paraît intéressant de comparer l'évolution de ces indices d'une campagne à l'autre avec les estimations absolues fournies à chaque fois par l'échointégration. Parallèlement, l'échantillonnage des zones littorales, non prospectées par l'hydroacoustique, à l'aide de filets verticaux permettrait d'estimer la quantité (ou tout au moins l'abondance relative) des poissons ignorés par le second procédé.

L'utilisation conjointe des deux approches offre donc un large éventail de perspectives pour une meilleure
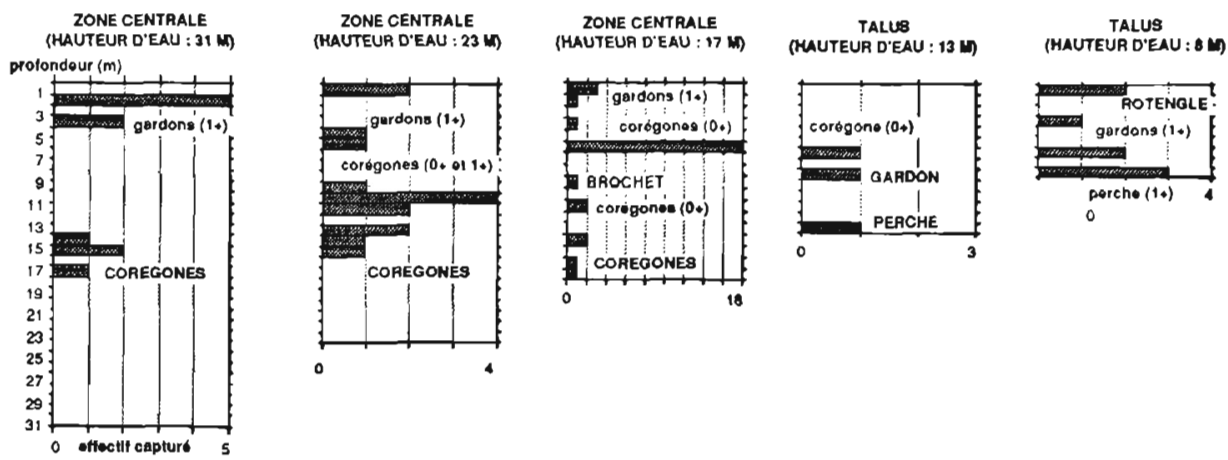

Fig. 8. - Répartition des captures réalisées durant un cycle de 24 heures par 5 batteries de filets verticaux disposées en zones centrales et sublittorales (llay, 24 juillet 1989).

Fig. 8. - Distribution of catches during a 24 hours cycles by 5 sets of vertical gill-nets held in central and sublittoral zone (llay lake; the 24th of July 1989). 
connaissance de l'ichtyofaune lacustre et pour l'élaboration d'une méthode synthétique fiable de suivi des peuplements lacustres. Une première opération combinant les deux techniques a été réalisée sur le lac StPoint, début octobre 1992.

\section{RÉFÉRENCES \\ BIBLIOGRAPHIQUES}

Allen K.R., 1966. Some Methods for Estimating Exploited Population. J. Fish. Res. Bd. Canada, 23(10) : 1553-1575.

Barbier B., 1985. Les technique de captures. Engins passifs: les filets maillants. Gestion piscicole des lacs et retenues artificielles, D. Gerdeaux et R. Billard ed., INRA Paris. : 81-90.

Chapman D.W., 1971. Production. Methods for Assessment of Fish Production in Freshwater. W.E. Ricker ed., 2nde ed., IBP handbook, 2 : 199-214.

CEEC, 1988. L'habitat lacustre et les peuplements pisciaires. Rappont S.R.E.T.I.E. : 52 pp.

Cury Ph., 1985. Note sur la production biologique et l'exploitation d'une population de poisson. Gestion piscicole des lacs et retenues artificielles, D. Gerdeaux et R. Billard ed., INRA Paris. : 147-155.

Degiorgi F. \& Grandmottet J.P., 1992. Impact de la désoxygénation chronique d'un lac de moyenne montagne sur son icthyofaune. Ichtyophysiologica Acta, 15 : 79-97.

Degiorgi F. \& Grandmottet J.P., 1993. Relation entre la topographie aquatique et l'organisation spatiale de l'ichtyofaune lacustre: définition des modalités spatiales d'une stratėgie de prélevement reproductible. Bull. Fr. de Pisc., 329 : 199-220.
Degiorgi F. \& Grandmottet J.P., soumis. Proposition d'un protocole standard d'échantillonnage de l'ichtyofaune lacustre fournissant une image comparable des peuplements. Bull. Fr. de Pisc.

Dembinsli W., 1971. Vertical distribution of vendace Coregonus albula $\mathrm{L}$. and other pelagic fish species in some Polish lakes. J. Fish. Biol., 3 : 341-357.

EIFAC 1975. Symposium sur les méthodes de prospection, de surveillance et d'évaluation des ressources ichtyologiques dans les lacs et les grands cours d'eau. FAO Tech. Pap. : 23.

Gerdeaux D. \& Guillard J., 1992. Utilisation des techniques acoustiques dans un fleuve: échosondage sur la Seine. Rapp. I. L., 65-92, 11 pp.

Gerdeaux D., 1986. Ecologie du Gardon (Rutilus rutilus) et du sandre (Lucioperca lucioperca L.) dans le lac de Créteil de 1977 à 1982. Etude de la ligulose du gardon. Th. Doct. Sc. Nat. univ. de Paris VI: $165 \mathrm{pp}$.

Gerdeaux D., 1985. Techniques d'échantillonnage. Les engins actifs : chalut et sennes. Gestion piscicole des lacs et retenues artificielles, D. Gerdeaux et R. Billard ed., INRA Paris. : 91-105.

Grandmottet J.P. \& Vaudaux P., 1989. Utilisation des filets verticaux pour l'échantillonnage des peuplements pisciaires : premiers résultats et perspectives. Actes du colloque de I'IGGE, Juin $89: 17$ pp.

Guillard J., Gerdeaux D., Brun G. \& Chapaz R., 1992. The use of geostatistic to analyse data from a echointegration survey of fish stock in lake SainteCroix. Fisheries research,13: 395406.

Guillard J., 1991. Etude des stock piscicoles par échointégration: problèmes méthodologiques. Th. Doct. Sc. Univ. Cl. Bernard, Lyon I: $156 \mathrm{pp}$.

Guyard A., Grandmottet J.P. \& Verneaux J., 1989. Utilisation de batteries de filets verticaux à enroulement : nouvelle 
technique d'échantillonnage de la faune ichtyologique lacustre. Application à l'étude du peuplement pisciaire de la retenue du barrage de Vouglans (Jura). Ann. Sci. Univ. Fr. Comté, Biol. anim., 5(1) : 59-70.

Hamley J.M., 1975. Review of gillnet selectivity. J. Fish. Res. Bd. Canada, 32(11) : 1943-1969.

Heland P., 1990. Problématique de la gestion piscicole: place de la recherche dans la conception d'une gestion rationnelle. Rev. des Sci. de l'Eau, 2(4): 793-806.

Helsert E. \& Condrey R.E., 1991. A new method of estimating gillnet selectivity, with an example for spotted seatrout, Cynocion nebulosus. Can. J. Fish. Aquat. Sc., $48:$ 487-492.

Horark D.L. \& Tanner H.A. The use of vertical gill nets in studying fish depth distribution. Trans. Am. Fish. Soc. 103(2) : 348-352.

Iste, 1992. Mise au point d'un protocole d'échantillonnage de l'ichtyofaune lacustre. Rapport scientifique réalisé pour le Conseil Supérieur de la Pêche : 20 pp.

Legendre P. \& Beauvais A., 1978. Niches et association de poissons des lacs de la Radissonie québecoise. Naturalist. can., 105 : 137-158.

Léopold M., Korulczyk T., Swierzowska L. \& Novak W., 1975. Effectiveness of seine catches for the estimation of fish populations in polish lakes. FAO, Eifac, 23(suppl.I), 48-57.
Lindem T., 1982. Successes with conventionnal in situ determination of fish target strength. Symposium on Fisheries Acoustics, Bergen, Norway, 2124 June 1982, contribution $n^{\circ} 53$ : $18 \mathrm{pp}$.

Marchal E., 1985. La détection acoustique dans l'étude des peuplements pisciaires. Gestion piscicole des lacs et retenues artificielles, D. Gerdeaux et R. Billard ed., INRA Paris :107-124.

MNEP, 1992. Etude piscicole de la retenue du Vieux Pré. Rapport EDF, 81 p.

Nelson R.W. \& Boussu F.M., 1974. Evaluation of trawls for monitoring and harvesting fish populations in lake Ohae South Dakota

Ricker W.E., 1980. Calcul et interprétration des statistiques biologiques des populations de poissons. Bull. Can. Sci. Lal. aq., Ottawa: 409 pp.

Savard L. \& Moreau G., 1981. Etude des relations entre les communautés piscicoles et les différents habitats d'une rivière nordique; notion d'habitat optimal. Can. J. of Zool., 60 : 3344-3352

Sandlund O.T., Nasje T.F., Klyve L. \& Lindem T., 1985. The vertical distribution of fish species in lake Mjøsa, Norway, as show by gill net catches and echo sounder. Rep. Inst. Freshwater Res. Drottningholm, 62 : 136-149.

Schnabel L.E., 1938. The estimation of the total fish population Lake. Am. Math. Month., 45: 348-353. 